\title{
The relationship between levels of self-esteem and the development of depression in young adults with mild depressive symptoms
}

\author{
Yoobin Choi ${ }^{1}$, Soo-Hee Choi ${ }^{1}$, Je-Yeon Yun²,3, Jae-A Lim¹, Yoonhee Kwon" \\ Hwa Young Lee ${ }^{4}$, Joon Hwan Jang ${ }^{4,5, *}$ \\ ${ }^{1}$ Department of Psychiatry, Seoul National University Hospital, Seoul, Republic of Korea. \\ 2 Seoul National University Hospital, Seoul, Republic of Korea. \\ ${ }^{3}$ Yeongeon Student Support Center, Seoul National University College of Medicine, Seoul, Republic of Korea. \\ ${ }^{4}$ Department of Psychiatry, Seoul National University Health Service Center, Seoul, Republic of Korea. \\ ${ }^{5}$ Department of Medicine, Seoul National University College of Medicine, Seoul, Republic of Korea.
}

\section{Introduction}

Self-esteem is defined as "a certain attitude and a perception of one's self", which affects interactions and feelings towards oneself and others. Little is known about the relationship between levels of self-esteem and the development of depression in young adults. The present study investigated the relationship between self-esteem and depression to determine whether self-esteem levels are a risk factor for the development of depression in young adults.

\section{Method}

This study was conducted with 113 college students aged 19 to 35 (Major Depressive Disorder (MDD) $n=44$, Mild Depressive Symptoms (MDS) $n=37$, Healthy Control $n=32$ ). The levels of clinical symptoms, self-esteem, resilience, social support, and quality of life, as well as personality traits, were assessed (by Patient Health Questionnaire-9(PHQ-9), Generalized Anxiety Disease-7(GAD-7), State-Trait Anxiety Inventory-S(STAI-S), Resilience Appraisal Scale(RAS), Rosenberg Self-Esteem Scale(RSES), Quality of Life, and NEO-Personality Inventory (NEO-PI)).

\section{Results}

There was no significant difference between the three groups in terms of sex and age. However, there were significant differences between the three groups in all clinical variable scores ( $p<0.01$ for all variables) (Table 1 ).
Table 1. The demographic and clinical characteristics

\begin{tabular}{lcccc}
\hline & $\begin{array}{c}\text { CON } \\
(\mathrm{N}=44)\end{array}$ & $\begin{array}{c}\text { MDS } \\
(\mathrm{N}=37)\end{array}$ & $\begin{array}{c}\text { MDD } \\
(\mathrm{N}=32)\end{array}$ & $\begin{array}{c}\text { Statistics } \\
\boldsymbol{p}\end{array}$ \\
\hline Sex (female/ male) & $27 / 17$ & $22 / 15$ & $19 / 13$ & 0.980 \\
Age (yrs) & $24.34 \pm 3.38$ & $24.78 \pm 3.49$ & $24.38 \pm 3.57$ & 0.827 \\
PHQ-9 & $3.50 \pm 5.05$ & $7.73 \pm 3.77$ & $10.38 \pm 2.51$ & $<0.001^{* *}$ \\
GAD-7 & $3.23 \pm 2.72$ & $5.92 \pm 4.72$ & $8.19 \pm 4.53$ & $<0.001^{* *}$ \\
STAI-S & $45.18 \pm 8.91$ & $53.54 \pm 8.83$ & $57.59 \pm 8.05$ & $<0.001^{* *}$ \\
RAS & $44.14 \pm 7.55$ & $37.03 \pm 5.81$ & $35.36 \pm 8.81$ & $<0.001^{* *}$ \\
RSES & $30.23 \pm 5.18$ & $25.68 \pm 4.61$ & $25.56 \pm 4.35$ & $<0.001^{* *}$ \\
Social Support & $97.07 \pm 13.04$ & $88.22 \pm 16.07$ & $85.88 \pm 11.44$ & $0.001^{*}$ \\
\hline *P $<05$ ** $\mathrm{P}<0.001$ & & & &
\end{tabular}

${ }^{*} \mathrm{P}<0.05$. $\quad{ }^{* *} \mathrm{P}<0.001$

The MDS group with high self-esteem reported having the lowest levels of social support, resilience, agreeableness and extraversion compared to those of the MDD group and control group with high self-esteem. In contrast, the MDS group with low self-esteem showed no differences in social support, resilience, agreeableness and openness according to the NEO-PI scale (Table 2).

\section{Conclusions}

Our findings suggest that a lack of social support may decrease resilience and lead to depression in young adults with high selfesteem. Early intervention for depression in young adults needs to focus on improving their levels of social support, resilience, and positive domains of personality.

Table 2. The mean scores and standard deviations of all clinical variables according to the level of self-esteem

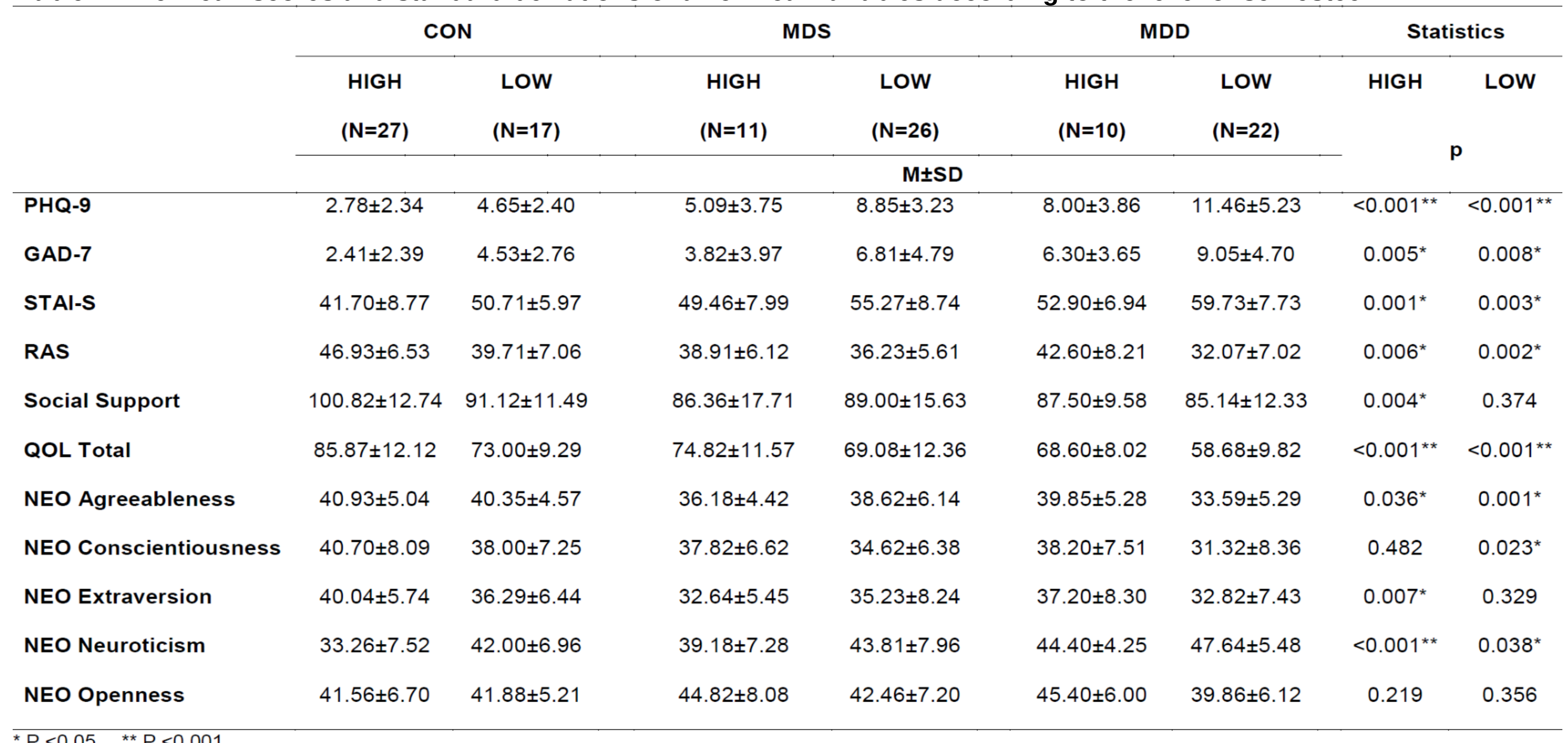

